\title{
Design of a Modified Tracking Differentiator
}

\author{
Zhigao Liu', Yaguang Jiang² \\ ${ }^{1}$ Department of Flight Control Technology, Beijing Aerospace Institute of Microsystems, Beijing, China \\ ${ }^{2}$ Department of Test Technology, China Software Testing Center, Beijing, China \\ Email: facth@163.com
}

How to cite this paper: Liu, Z.G. and Jiang, Y.G. (2017) Design of a Modified Tracking Differentiator. World Journal of Engineering and Technology, 5, 668-674. https://doi.org/10.4236/wjet.2017.54055

Received: August 25, 2017

Accepted: October 30, 2017

Published: November 2, 2017

Copyright $\odot 2017$ by authors and Scientific Research Publishing Inc. This work is licensed under the Creative Commons Attribution International License (CC BY 4.0).

http://creativecommons.org/licenses/by/4.0/

\begin{abstract}
A modified tracking differentiator is proposed. Firstly, a nonlinear odd exponent continuous function is adopted which is only stable at one equilibrium point and proved the global asymptotic stability of the modified tracking differentiator by select a Lyapunov function. Through combining of the nonlinear and linear function properly, it can be sure that the state converges to the equilibrium point with high speed automatically no matter that the state was far away from the equilibrium point or near to it, and it can prevent the chattering. Simulation results show that the modified tracking differentiator tracking results are superior to the classical nonlinear tracking differentiator, and the response of state variables tracking differentiator estimated is almost coincide with the real state of the variables of the given system.
\end{abstract}

\section{Keywords}

Tracking Differentiator, Lyapunov Function, Nonlinear, Switch Function, Chattering

\section{Introduction}

In actual engineering, it is often necessary to extract the differential signal from a given signal, and it is important to obtain accurate differential signal for the controller design, especially for the design of modern control theory based on the exact mathematical model. Usually a first order inertial component or difference method is used to obtain differential signals. But when the signal contains noise, the noise can be amplified when the differential signal is obtained with the above two methods (general noise change rate is better than the useful signal changes rate). In particular, a noise differential signal is obtained, and even the differential signal of the desired signal is drowned. In the literature 1, a second-order fastest switching system is used to construct a mechanism to extract the approximate differential signal, and the concept of the nonlinear track- 
ing differentiator is proposed. The general form of the nonlinear tracking differentiator is given. The linear tracking differentiator changes the differential equation before the first-order inertia or differential method, and can better track the differential signal of a given signal [1]. However, the method of the literature 1 contains the switching function, which is not conducive to the system proof and stability analysis [2] [3]. According to the literature 2, the exponential of the function of the nonlinear tracking differential in the literature 1 is expressed by the fractional form of the odd number of numerator and denominator, which is replaced by the form of the switching function. The conclusion is that the output signal can track the input signal point by point when the time scale tends to be positive. The advantage of this method is that the convergence speed is faster near the equilibrium point. The disadvantage is that when the system state is far from the equilibrium point, the convergence speed is slower, which leads to the serious chattering of the tracking curve in the initial stage [3] [4] [5]. In the literature 3, a full range fast nonlinear tracking differentiator is designed, which automatically converges at a faster rate when the system state is far from or near equilibrium. Although this design has achieved beneficial results, due to the use of nonsmooth fractional exponential functions near the poles of the system, the tracking signal has shown pronounced tremors [5] [6]. Although the tracking differentials designed in the literature 4 and literature 5 have reduced this tremor, the designed method is prone to produce complex solutions, and its solution on the imaginary axis makes the tracer system in a critical stable state, which may lead to the system is unstable or the output has a greater tremor [6] [7] [8] [9] [10].

In order to improve the performance of the tracking differentiator, it is necessary to remove the complex solution of the tracer, in particular to remove the solution on the imaginary axis, and to satisfy the requirement that the tracking differentiator converge quickly [10] [11] [12] [13] [14]. This paper designs an improved tracking differentiator. The Lyapunov theorem is used to prove the stability of the improved tracer, and the theoretical analysis and simulation prove that it can accelerate the convergence when it is away from the equilibrium point and close to the equilibrium point.

\section{Design of Improved Tracking Differentiator}

Assuming that for system $S_{1}[1][7]$

$$
\left\{\begin{array}{l}
\dot{x}_{1}(t)=x_{2}(t) \\
\dot{x}_{2}(t)=f\left[x_{1}(t), x_{2}(t)\right]
\end{array}\right.
$$

where $X(t)=\left[x_{1}(t), x_{2}(t)\right]$ has the following theorem:

Theorem 1: For system $S_{1}$, if its solution $x_{1}(t), x_{2}(t)$ satisfies: $\lim _{t \rightarrow \infty} x_{1}(t)=0$, $\lim _{t \rightarrow \infty} x_{2}(t)=0$, then there will be system $S_{2}$

$$
\left\{\begin{array}{l}
\dot{x}_{1}(t)=x_{2}(t) \\
\dot{x}_{2}(t)=R^{2} \cdot f\left[\left(x_{1}(t)-v(t)\right), \frac{x_{2}(t)}{R}\right]
\end{array}\right.
$$


For any given bounded integrable function $v(t)$ and constant $T$, the solution of Equation (2) satisfies: $\lim _{t \rightarrow \infty} \int_{0}^{T}\left|x_{1}(t)-v(t)\right| \mathrm{d} t=0$ (or $\left.\lim _{t \rightarrow \infty} \int_{0+t_{0}}^{T+t_{0}}\left|x_{1}(t)-v(t)\right| \mathrm{d} t=0\right), \lim _{t \rightarrow \infty}^{t \rightarrow \infty} x_{2}(t)=0$. Proof process see the literature 1.

The key to selecting the differential tracker is to select $f\left[x_{1}(t), x_{2}(t)\right]$, which is set here as follows:

$$
\dot{x}_{2}(t)=f\left[x_{1}(t), x_{2}(t)\right]
$$

Equal

$$
\dot{x}_{n}(t)=-a_{1} x_{1}(t)-b_{1} x_{2}(t)-b_{2} x_{2}(t)^{n}
$$

The following theorems are obtained:

Theorem 2: The system $S_{3}$

$$
\left\{\begin{array}{l}
\dot{x}_{1}(t)=x_{2}(t) \\
\dot{x}_{2}(t)=-a_{1} x_{1}(t)-b_{1} x_{2}(t)-b_{2} x_{2}(t)^{n}
\end{array}\right.
$$

When $a_{1}>0, b_{1}>0, b_{2}>0, n>0$, and $n$ is an odd number, the solution of the Formula (5) satisfies the conclusion of the system $S_{1}$ in theorem 1, and the system $S_{3}$ is stabilized and stabilized at $\left(x_{1}, x_{2}\right)^{\mathrm{T}}=(0,0)^{\mathrm{T}}$, that is $\lim _{t \rightarrow \infty} x_{1}(t)=0$, $\lim _{t \rightarrow \infty} x_{2}(t)=0$.

The proof of theorem 2 is as follows:

Select the Lyapuvov energy function $V$, so $V=a_{1} x_{1}(t)^{2} / 2+x_{2}(t)^{2} / 2+1$, because $a_{1}$ is greater than 0 , then the function $V$ coefficient is greater than 0 , and because $x_{i}(t)^{2} \geq 0,(i=1,2)$, so for any state $\left(x_{1}, x_{2}\right)^{\mathrm{T}}$ have $V=a_{1} x_{1}(t)^{2} / 2+x_{2}(t)^{2} / 2+1>0$.

The derivative of $V$ on both sides is obtained:

$$
\begin{gathered}
\dot{V}=\frac{\partial V}{\partial x_{1}} \dot{x}_{1}(t)+\frac{\partial V}{\partial x_{2}} \dot{x}_{2}(t) \\
\dot{V}=\frac{\partial V}{\partial x_{1}} x_{2}(t)+\frac{\partial V}{\partial x_{2}}\left[-a_{1} x_{1}(t)-b_{1} x_{2}(t)-b_{2} x_{2}(t)^{n}\right]
\end{gathered}
$$

Also because:

$$
\begin{gathered}
\frac{\partial V}{\partial x_{1}}=a_{1} x_{1}(t) \\
\frac{\partial V}{\partial x_{2}}=x_{2}(t)
\end{gathered}
$$

The Formula (8) and the Formula (9) are given to the Formula (7):

$$
\dot{V}=a_{1} x_{1}(t) x_{2}(t)+x_{2}(t)\left[-a_{1} x_{1}(t)-b_{1} x_{2}(t)-b_{2} x_{2}(t)^{n}\right]
$$

Finished:

$$
\dot{V}=-b_{1} x_{2}(t)^{2}-b_{2} x_{2}(t)^{n+1}
$$

Because $b_{1}, b_{2}, n$ are greater than 0 , the function $\dot{V}$ of the coefficient are less than 0 , and because $\mathrm{v}$ is greater than 0 odd, it is $\mathrm{x}$, so for any state $\mathrm{x}$ have $\mathrm{v}$. That is, the system is gradual and stable, that can eventually think that $\mathrm{x}$ tends to 
a certain stability does not change, then this time there must be $\mathrm{x}$. Because $\mathrm{xx}$, there $\mathrm{xxx}$.

Since $b_{1}, b_{2}, n$ are greater than 0 , the coefficients of function $\dot{V}$ are less than 0 , and because $n$ is odd number greater than 0 , there is $x_{2}(t)^{n+1} \geq 0$, so $\left(x_{1}, x_{2}\right)^{\mathrm{T}}$ for any state has $\dot{V}=-b_{1} x_{2}(t)^{2}-b_{2} x_{2}(t)^{n+1} \leq 0$. That is, the system is asymptotically stable that can eventually think that $x_{1}(t)$ tends to a certain stability does, then this time there must be $\dot{x}_{1} \equiv 0, x_{2}=\dot{x}_{1} \equiv 0$. Because $x_{2} \equiv 0$, there $\dot{x}_{2} \equiv 0$.

$x_{2} \equiv 0, \quad \dot{x}_{2} \equiv 0 \quad$ are given to the Formula (5):

$$
-a_{1} x_{1}(t)=0
$$

Answer is:

$$
x_{1}(t)=0
$$

That is, the system stability value is:

$$
\left(x_{1}, x_{2}\right)^{\mathrm{T}}=(0,0)^{\mathrm{T}}
$$

So far, theorem 2 is proved.

According to theorem 1 and theorem 2, the form of an improved tracking differentiator can be obtained, as shown in Equation (15):

$$
\left\{\begin{array}{l}
\dot{x}_{1}(t)=x_{2}(t) \\
\dot{x}_{2}(t)=-R^{2}\left\{a_{1}\left[x_{1}(t)-v(t)\right]-b_{1} \frac{x_{2}(t)}{R}-b_{2} \frac{x_{2}(t)^{n}}{R^{n}}\right\}
\end{array}\right.
$$

In Formula (15), $a_{1}>0, b_{1}>0, b_{2}>0, n>0, n$ is odd.

For any given bounded integrable function $v(t)$ and constant $T$, the solution of Equation (15) satisfies: $\lim _{t \rightarrow \infty} \int_{0}^{T}\left|x_{1}(t)-v(t)\right| \mathrm{d} t=0 \quad$ (or $\left.\lim _{t \rightarrow \infty} \int_{0+t_{0}}^{T+t_{0}}\left|x_{1}(t)-v(t)\right| \mathrm{d} t=0\right), \lim _{t \rightarrow \infty} x_{2}(t)=0$.

It is necessary to make the following 3 points for the improved tracking differentiator shown in Equation (15):

1) For Equation (15), when $e=x_{1}(t)-v(t)$ is large, the acceleration signal $\dot{x}_{1}(t)$ (or $x_{2}(t)$ ) will be larger, so that the system can be faster to track $v(t)$, to meet $\lim _{t \rightarrow \infty} \int_{0+t_{0}}^{T+t_{0}}\left|x_{1}(t)-v(t)\right| \mathrm{d} t=0$. At this time, $b_{1} \frac{x_{2}(t)}{R}<b_{2} \frac{x_{2}(t)^{n}}{R^{n}}, b_{2} \frac{x_{2}(t)^{n}}{R^{n}}$ plays a major role in making $\left(x_{1}-v, x_{2}\right)^{\mathrm{T}} \rightarrow(0,0)^{\mathrm{T}}$, accelerating the $v(t)$ process.

2) When $e=x_{1}(t)-v(t)$ is small, that is, when $x_{1}(t)$ is close to $v(t)$, the $x_{1}(t)$ changes slowly, that is, the slope becomes smaller, the acceleration signal $\dot{x}_{1}(t)$ (or $x_{2}(t)$ ) becomes smaller, and then the acceleration is still needed as much as possible, so that the system can track the $v(t)$ at a relatively fast speed, and meet $\lim _{t \rightarrow \infty} \int_{0+t_{0}}^{T+t_{0}}\left|x_{1}(t)-v(t)\right| \mathrm{d} t=0$.

At this time, just $b_{1} x_{2}(t) / R>b_{2} x_{2}(t)^{n} / R^{n}$,

(At this time $\left(x_{1}-v, x_{2}\right)^{\mathrm{T}} \rightarrow(0,0)^{\mathrm{T}}, b_{2} x_{2}(t)^{n} / R^{n} \quad$ slope close to the $\mathrm{x}$-axis), $b_{2} x_{2}(t) / R$ play a major role. 
As a result of the synthesis (1) (2), the improved tracking differential is designed so that the state quantity can accelerate convergence when it is away from the equilibrium point and near the equilibrium point.

3) If: $f=-a_{1} x_{1}(t)-a_{2} x_{1}(t)^{m}-b_{1} x_{2}(t)-b_{2} x_{2}(t)^{n}$, is it possible to ensure that the formula 15 in $e=x_{1}(t)-v(t)$ larger, in addition to relying on $b_{2} x_{2}(t)^{n} / R^{n}$ but also rely on non-linear link $-a_{2} x_{1}(t)^{m}$ accelerated tracking $v(t)$ process, the system can be faster to track $v(t)$, to meet $\lim _{t \rightarrow \infty} \int_{0+t_{0}}^{T+t_{0}}\left|x_{1}(t)-v(t)\right| \mathrm{d} t=0$ ? The answer is negligible. From the theoretical calculation we can see that $\left(x_{1}, x_{2}\right)^{\mathrm{T}}$ is convergent to $(0,0)^{\mathrm{T}}$, but also converges to $\left[\left(-a_{1} / a_{2}\right)^{1 /(m-1)}, 0\right]^{\mathrm{T}}$ (where $a_{1}, a_{2}, m$ is greater than 0 and $m$ is positive odd, then $\left(-a_{1} / a_{2}\right)^{1 /(m-1)}$ is a complex solution, which is located on the imaginary axis, resulting in critical stability of the tracer system, If we prove that the optional Lyapuvov energy function $V, V=a_{1} x_{1}(t)^{2} / 2+a_{2} x_{1}(t)^{m+1} /(m+1)+x_{2}(t)^{2} / 2$, can prove that the two solutions converged by the system are $x_{1}(t)=0, \quad x_{1}(t)=\left(-a_{1} / a_{2}\right)^{1 /(m-1)}$, detailed proof ).

\section{Improved Tracking Differentiator Simulation}

In the simulation, the improved tracking differential is compared with the classical nonlinear differential splitter designed in literature 1. The parameters for the non-linear tracking differentiator are set as follows: The TD setting of ADRC [1]: $\mathrm{r}=12, \mathrm{~d}=0.05$.

The parameters for the improved tracking differentiator are set as follows: $R=$ $5, a=5, b_{1}=b_{2}=1, n=3$.

The simulation results show that the improved tracking differentiator has stable tracking effect, and the chattering is small in Figure 1 and Figure 2 . At the same time, the tracking of the differential signal value is larger, in line with the step signal changes in the moment, the rate of change is extremely high. It can be seen from Figure 3 that when the differential signal of sinusoidal signal is estimated, the improved tracking differentiator is better than the TD-ADRC.

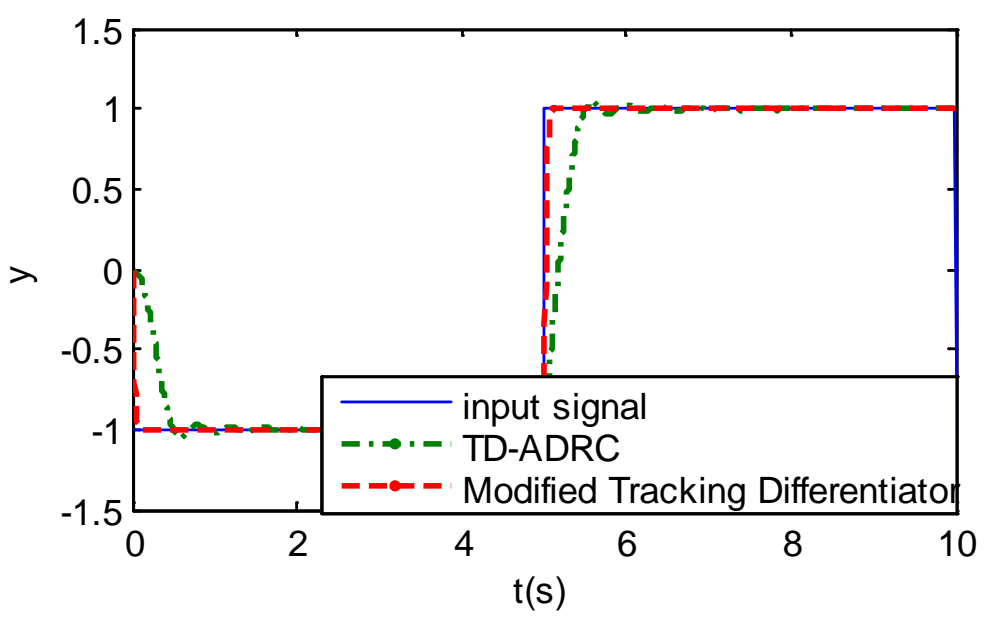

Figure 1. Comparison of the effects of two tracking differentiator tracking input values. 


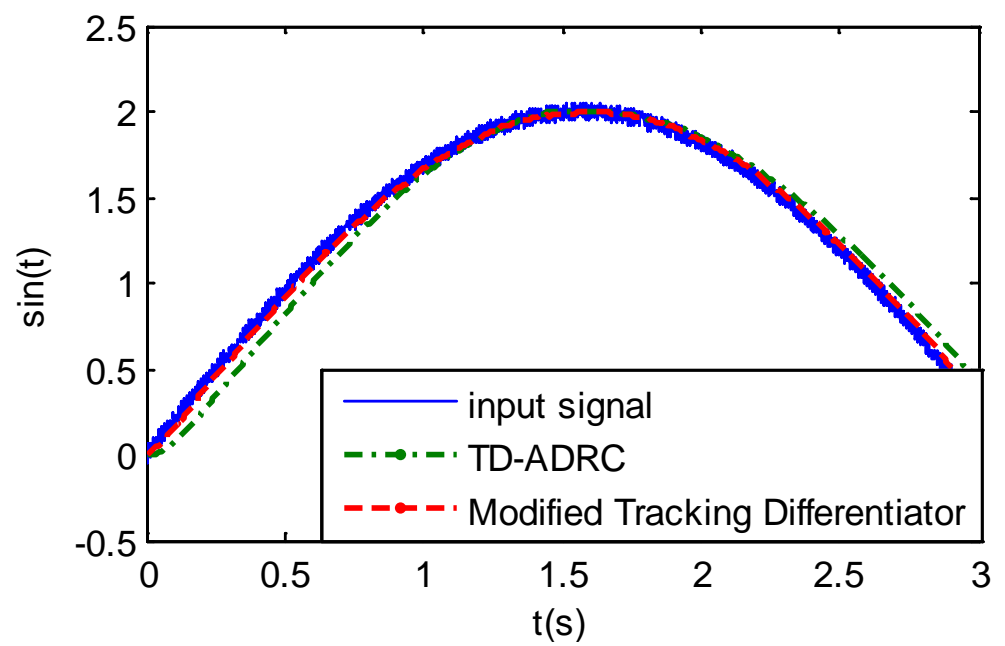

Figure 2. Comparison of the effects of two differentiator tracer estimation differential signals.

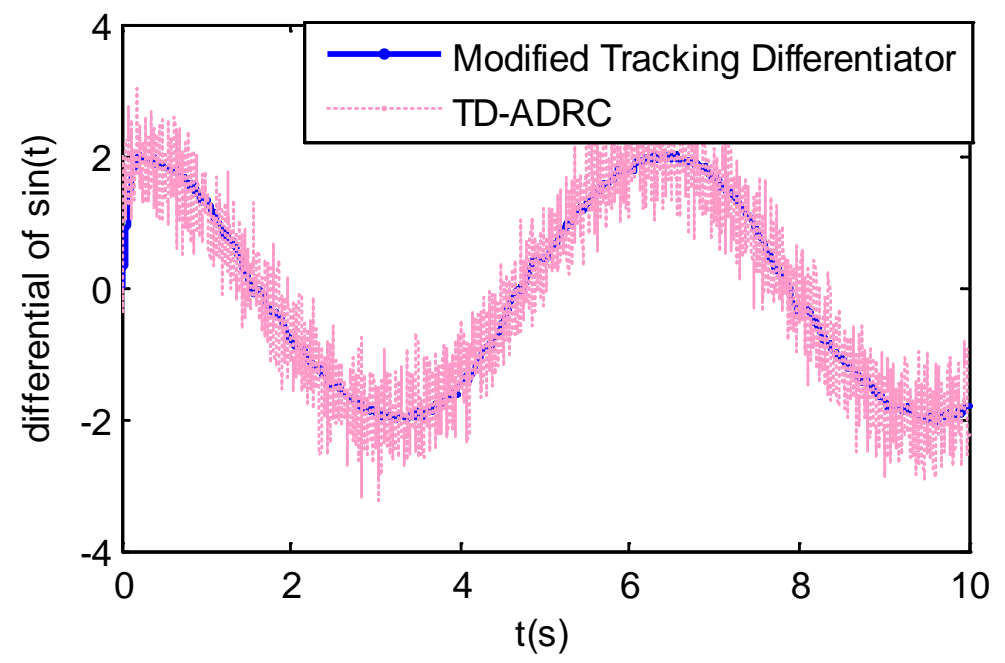

Figure 3. Comparison of Sine differential signal.

\section{Conclusion}

The improved tracking differentiator designed in this paper is a stable system in the sense of Lyapunov, which has global stability and fast convergence. Through simulation, comparison and verification, it is proved that the improved tracking differentiator has good tracking effect, and it has simple form and no chattering when tracking. It is a better tracking differentiator.

\section{References}

[1] Han, J.Q. and Wang, W. (1994) Nonlinear Tracking Differ Entiator. Journal of System \& Mathematic Science, 14, 177-183.

[2] Wang, X.H. and Chen, Z.Q. (2003) Design and Stability Analysis for a New Nonlinear Tracking-Differentiator. The 21 th Chinese Control Conf. Zhejiang University Press, Hangzhou, 64-68.

[3] Wang, X.H., Chen, Z.Q. and Yuan, Z.Z. (2003) Nonlinear Tracking Differentiator 
with High Speed in Whole Course. Control Theory \& Applications, 20, 875-878.

[4] Zhao, P., Yao, M.L., Lu, C.J. and Hu, Y.T. (2011) Design of Nolinear Linear Tracking Differentiator with High Stability and High Speed. Journal of Xi An Jiao Tong University, 45, 43-48.

[5] Shi, Y.L. and Hou, C.Z. (2008) Design of Improved Nonlinear Tracking Differentiator. Control and Decision, 23, 647-650.

[6] An, J.Q. (2001) Nonlinear PID Controller. Acta Automatica Sinica, 20, 487-489.

[7] Peng, J.L., Sun, X.X., Dong, W.H., et al. (2001) Constructing the Maneuvering Target Estimation Model with Tracking Differentiator. Flight Dynamics, 28, 59-63.

[8] Zhang, H.B., Huang, X.H., Wang, M., et al. (2012) Precise Control of Linear Systems Subject to Actuator Saturation Using Tracking Differentiator and Reduced Order Composite Nonlinear Feedback Control. International Journal of Systems Science, 43, 220-230. https://doi.org/10.1080/00207721.2010.488757

[9] Guo, B.-Z. and Zhao, Z.-L. (2011) On Convergence of Tracking Differentiator. International Journal of Control, 84, 693-701. https://doi.org/10.1080/00207179.2011.569954

[10] Wang, J.H., Zhang, J.L. and Yan, J. (2010) A New Second Order Nonlinear Tracking Differentiator and Application. 2010 International Conference on Computer Design and Applications (ICCDA), V1-318-V1-322.

[11] Qiao, G.H., Sun, M.L. and Zhang, R. (2009) An Improved Self-Adaptive Tracking Differentiator. International Conference on Information and Automation, 2009. ICIA '09. 152-156.

[12] Zhu, J.H., Zhang, Z.J. and Yang, H.Z. (2006) An Extended State Observer Based on Tracking Differentiator. Control Conference, CCC 2006. Chinese 2006, 86-89.

[13] Dong, X.-M. and Zhang, P. (2001) Design and Phase Plane Analysis of an Arctangent-Based Tracking Differentiator. Control Theory \& Applications, No. 4, 129-133.

[14] Wang, Q.L. and Jiang, Z.R. (1999) Linear Tracking Differentiators and Its Applications in State Feedback Control. Journal of Beijing Institute of Technology, No. 2, 75-78. 